\title{
Telomere profiles and tumor-associated macrophages with different immune signatures affect prognosis in glioblastoma
}

\author{
Noelyn A Hung ${ }^{1}$, Ramona A Eiholzer ${ }^{1}$, Stenar Kirs ${ }^{2}$, Jean Zhou ${ }^{3}$, Kirsten Ward-Hartstonge ${ }^{4}$, \\ Anna K Wiles ${ }^{1}$, Chris M Frampton ${ }^{5}$, Ahmad Taha ${ }^{6}$, Janice A Royds ${ }^{1}$ and Tania L Slatter ${ }^{1}$ \\ ${ }^{1}$ Department of Pathology, Dunedin School of Medicine, University of Otago, Dunedin, New Zealand; \\ ${ }^{2}$ Department of Surgical Sciences, Dunedin School of Medicine, University of Otago, Dunedin, New Zealand; \\ ${ }^{3}$ Department of Radiology, Southern District Health Board, Dunedin, New Zealand; ${ }^{4}$ Department of \\ Microbiology and Immunology, Dunedin School of Medical Sciences, University of Otago, Dunedin, New \\ Zealand; ${ }^{5}$ Department of Medicine, University of Otago, Christchurch, New Zealand and ${ }^{6}$ Neurosurgery, \\ Southern District Health Board, Dunedin, New Zealand
}

\begin{abstract}
Telomere maintenance is a hallmark of cancer and likely to be targeted in future treatments. In glioblastoma established methods of identifying telomerase and alternative lengthening of telomeres leave a significant proportion of tumors with no defined telomere maintenance mechanism. This study investigated the composition of these tumors using RNA-Seq. Glioblastomas with an indeterminate telomere maintenance mechanism had an increased immune signature compared with alternative lengthening of telomeres and telomerase-positive tumors. Immunohistochemistry for CD163 confirmed that the majority $(80 \%)$ of tumors with an indeterminate telomere maintenance mechanism had a high presence of tumor-associated macrophages. The RNA-Seq and immunostaining data separated tumors with no defined telomere maintenance mechanism into three subgroups: alternative lengthening of telomeres like tumors with a high presence of tumor-associated macrophages and telomerase like tumors with a high presence of tumor-associated macrophages. The third subgroup had no increase in tumor-associated macrophages and may represent a distinct category. The presence of tumorassociated macrophages conferred a worse prognosis with reduced patient survival times (alternative lengthening of telomeres with and without macrophages $P=0.0004$, and telomerase with and without macrophages $P=0.013$ ). The immune signatures obtained from RNA-Seq were significantly different between telomere maintenance mechanisms. Alternative lengthening of telomeres like tumors with macrophages had increased expression of interferon-induced proteins with tetratricopeptide repeats (IFIT1-3). Telomerase-positive tumors with macrophages had increased expression of macrophage receptor with collagenous structure (MARCO), CXCL12 and sushi-repeat containing protein x-linked 2 (SRPX2). Telomerase-positive tumors with macrophages were also associated with a reduced frequency of total/near total resections $(44 \% v s>76 \%$ for all other subtypes, $P=0.014$ ). In summary, different immune signatures are found among telomere maintenance mechanism-based subgroups in glioblastoma. The reduced extent of surgical resection of telomerase-positive tumors with macrophages suggests that some tumor-associated macrophages are more unfavorable. Modern Pathology (2016) 29, 212-226; doi:10.1038/modpathol.2015.156; published online 15 January 2016
\end{abstract}

With a median survival of approximately 14 months and death almost certain within 2 years of diagnosis the prognosis from glioblastoma is poor. ${ }^{1}$ In 2005 an amendment to the standard therapy of surgery and

Correspondence: Dr TL Slatter, PhD, Department of Pathology, Dunedin School of Medicine, University of Otago, PO Box 913, Dunedin 9054, New Zealand.

E-mail: tania.slatter@otago.ac.nz

Received 6 September 2015; revised 18 November 2015; accepted 20 November 2015; published online 15 January 2016 radiotherapy was recommended to include the oral alkylating agent temozolomide based on improved survival, particularly 2-year survival. ${ }^{1}$ Future therapies may target a key hallmark of cancerous cells, the telomere maintenance mechanism utilized to maintain limitless proliferation. Approximately $34-47 \%$ of glioblastoma maintain telomere lengths using telomerase activity. ${ }^{2-4}$ Telomerase inhibitors decrease glioma cell growth in vitro and in xenograft models, suggesting telomerase inhibitors may work in a clinical setting. ${ }^{5,6}$ 
The alternative lengthening of telomeres method also maintains telomeres and is found in approximately $11-25 \%$ of glioblastoma. ${ }^{2,3,7}$ Although the exact mechanism that gives rise to alternative lengthening of telomeres is unknown, distinguishing features are long and heterogeneous telomere lengths, and nuclear co-localizations of telomere DNA and the promyelocytic leukemia protein called alternative lengthening of telomere-associated promyelocytic leukemia bodies. $^{8-11}$ Alternative lengthening of telomere-positive tumors are associated with an improved patient survival, despite alternative lengthening of telomere-positive tumors responding poorly to adjuvant therapy. ${ }^{12}$ Tumors that are alternative lengthening of telomeres positive are associated with isocitrate dehydrogenase 1 (IDH1) and tumor protein 53 (TP53) mutations, a younger age, and secondary glioblastoma suggesting these tumors fit into the gene expression-based proneural subtype. ${ }^{2-4,12-15}$

For a large proportion of glioblastomas (40\%) the telomere maintenance mechanism is not known and they have been categorized as having a non-defined telomere maintenance mechanism..$^{3,4}$ It is possible that these tumors do not activate a telomere maintenance mechanism similar to low-grade astrocytomas, ${ }^{16,17}$ or that their telomere maintenance mechanism remains undescribed. ${ }^{18-20}$ Another possibility is that non-defined telomere maintenance mechanism tumors are comprised of telomerase and alternative lengthening of telomere-positive tumors, but complications in assaying telomerase activity and measuring the hallmarks of the alternative lengthening of telomeres mechanism lead to falsenegative results. Many glioblastomas contain a considerable proportion of non-neoplastic cells such as macrophages/microglia (tumor-associated macrophages) that can comprise over $70 \%$ of the tumor. ${ }^{21-25}$ Interference of tumor-associated macrophages is illustrated by the improvement in interpretation of MGMT (O-6-methylguanineDNA methyltransferase) status of glioblastoma using immunohistochemistry. ${ }^{26}$

That tumor-associated macrophages obscure the telomere maintenance mechanism is suggested by molecular similarities between glioblastomas classified as having the non-defined telomere maintenance mechanism and those typed as alternative lengthening of telomeres or telomerase positive. Many non-defined telomere maintenance mechanism tumors share similarities with telomerase-positive tumors. The non-defined telomere maintenance mechanism subgroup had a high percentage $(71 \%)$ that were positive for the embryonic marker paired boxed transcription factor 8 (PAX8) similar to that found in telomerase-positive tumors. ${ }^{27,28}$ In vitro PAX8 upregulates the expression of a key protein in telomerase, hTERT, which increases telomerase activity in glioma cells suggesting $P A X 8$ is associated with telomerase-positive glioblastoma. ${ }^{29}$ Telomerase and non-defined telomere maintenance mechanism tumors share other similarities including a similar median patient age, similar overall survival that is poorer compared with alternative lengthening of telomere-positive tumors and infrequent TP53 and IDH1 mutations. ${ }^{2-4}$ Investigated as a single group of non-alternative lengthening of telomeres tumors, non-defined telomere maintenance mechanism and telomerase-positive tumors responded to temozolomide. ${ }^{12}$ If non-defined telomere maintenance mechanism tumors are similar to telomerasepositive tumors they may respond to similar therapies such as telomerase inhibitors.

Some glioblastomas with a non-defined telomere maintenance mechanism share properties with alternative lengthening of telomeres tumors, in that they are positive for alternative lengthening of telomere-associated promyelocytic leukemia bodies despite not having clear long and heterogeneous telomere lengths. This suggests that some of the non-defined telomere maintenance group is comprised of tumors with prealternative lengthening of telomeres or masked-alternative lengthening of telomeres phenotype. ${ }^{16}$ In addition, non-defined telomere maintenance mechanism tumors with TP53 mutations showed a trend toward increased patient survival. ${ }^{2}$ Improved patient survival associated with TP53 mutations was found for alternative lengthening of telomeres tumors, but TP53 mutations in telomerasepositive tumors were associated with poor patient survival. ${ }^{2}$

In combination with RNA-Seq we report that glioblastomas with a non-defined telomere maintenance mechanism are comprised of three distinct subgroups, each with a poor patient prognosis. Two of these subgroups have high infiltrates of tumorassociated macrophages.

\section{Materials and methods}

\section{Tumor Samples}

Brain tumors were obtained from patients who attended neurosurgical units in Dunedin and Christchurch in New Zealand from 2002 to 2012. One hundred and thirty-six tumors were selected from the New Zealand brain tumor study cohort. The selection criteria were a diagnosis of glioblastoma, the first tumor obtained, patients who had surgery only, surgery and radiotherapy, or surgery, radiotherapy and temozolomide (completed course of six cycles of adjuvant treatment), and patients with completed clinical information. The cohort was biased toward alternative lengthening of telomerespositive tumors, in which all alternative lengthening of telomeres-positive tumors from a total cohort of 300 glioblastomas were included and 107 non-defined telomere maintenance mechanism and telomerase tumors were chosen at random from the total cohort. National ethics committees approved 
the study (MEC/08/02/016/AM01) and all patients provided written informed consent.

The criterion for an alternative lengthening of telomeres-positive tumor was the presence of alternative lengthening of telomeres-associated promyelocytic leukemia bodies plus long heterogeneous telomere lengths using the terminal restriction fragment length assay $(n=29)$. Telomerase-positive tumors included 50 cases that were positive for telomerase enzyme activity by the telomeric repeat amplification protocol assay. The non-defined telomere maintenance mechanism group comprised of 61 cases. All non-defined telomere maintenance mechanism tumors were negative for telomerase activity using the telomeric repeat amplification protocol assay criteria and did not have long and heterogeneous telomere lengths. Nine were positive and 52 negative for alternative lengthening of telomeres-associated promyelocytic leukemia bodies.

\section{Immunohistochemistry}

Tissue sections from paraffin-embedded material were subjected to heat-mediated antigen retrieval. The CD163 and PAX8 staining was performed twice so that two different antibodies were used: CD163 (EPR14643, Abcam, Cambridge, UK; and Novocastra 10D6; Leica Biosystems, Wetzlar, Germany) and PAX8 (MRQ-50; Cell Marque, Rocklin, CA, USA and BC12; Biocare Medical, Concord, CA, USA). MGMT staining used the MT3.1 primary antibody (Abcam). Positive cells were detected using EDL (Dako, Glostrup, Denmark) and DAB. For PAX8 and MGMT staining positive cells were counted in at least 10 high-powered fields $(\times 400$ magnification) using the Aperio Scancope CS digital pathology system (Aperio, Vista, CA, USA), and the percentage of positive cells per total cells was measured.

For CD163 staining two sections from two tissue blocks per tumor were analyzed by three blinded examiners. Stained slides were evaluated using the Aperio Scancope CS digital pathology system (Aperio), and the percentage of positive cells per total cells was measured. Eleven fields at random were chosen at $\times 400$ magnification, and the percentage of CD163 cells was measured per field. The fields with the lowest and highest percentages were eliminated and the median percentage of macrophages from the remaining fields was given a score $(0-3)$ based on the percent positive cells: $0(0-15 \%)$, $1(16-20 \%), 2(21-35 \%)$, and $3(>35 \%)$. Tumors with a high tumor-associated macrophage content were defined as those with a score of 3 on both tissue sections from both antibodies and from all examiners. Tumors with a low tumor-associated macrophage content were defined as those with a score of 0-2 on both tissue sections from all examiners. For equivocal cases a third tissue section from a third tissue block was analyzed and the median score from the three sections was used. For equivocal cases without a third tissue block the score representing the mean percentage of positive cells was used.

For PAX8 staining a positive tumor was defined as a tumor with $>30 \%$ of positive cells. ${ }^{28}$ MGMT was investigated using double staining alongside CD163 staining and used the MACH 2 Double Stain 2 Kit (Biocare Medical). The alkaline phosphatase secondary antibody was detected using Fast Red (Biocare Medical) and the HRP labeled secondary was labeled using DAB. MGMT-positive tumors had $>50 \%$ of malignant cells MGMT positive. At least three independent examiners (NH (anatomical pathologist), RE, SK, KH or TS) scored each slide in a blinded manner.

\section{Alternative Lengthening of Telomeres-Associated Promyelocytic Leukemia Body Measurement}

Measurement of co-localized bodies of the promyelocytic leukemia protein and telomere DNA, in cellular nuclei, was performed on paraffinembedded tumors according to the alternative lengthening of telomeres-associated promyelocytic leukemia body assay described previously. ${ }^{4,9}$ Briefly the promyelocytic leukemia protein was detected using a goat antipromyelocytic leukemia antibody (N-19; Santa Cruz Biotechnology, Santa Cruz, CA, USA) and telomere DNA was detected by fluorescence in situ hybridization with a Cy3-labeled PNA probe (Applied Biosystems, Framingham, MA, USA). Cellular nuclei were stained using 4,6-diamidino-2-phenylindole. Cells were imaged using confocal microscopy (Zeiss LSM510; Carl Zeiss, Thornwood, NY, USA), and images were analyzed by Zeiss LSM Image Examiner software version 3.2.0.115 (Carl Zeiss). Tumors were alternative lengthening of telomeres-associated promyelocytic leukemia body positive when co-localized promyelocytic leukemia protein/telomeres-DNA fluorescent signals were present in at least $5 \%$ of cells. Criteria for alternative lengthening of telomere-associated promyelocytic leukemia bodies also included a large size $(>0.9 \mu \mathrm{m})$ and co-localizations in sharp focus with a clear peak signal. ${ }^{4,9}$

\section{Telomere Length and Telomerase Activity Analyses}

Telomerase activity in tumors was detected using the telomerase repeat amplification protocol PCR based assay, ${ }^{4,30}$ and used the TeloTAGGG Telomerase PCR ELISA Plus Kit (Roche Applied Science, Mannheim, Germany) according to the manufacturer's instructions. The analysis of telomere length was made by the terminal restriction fragment length assay and used the TeloTAGGG Telomere Length Assay Kit (Roche Applied Science) according to the manufacturer's instructions. The criteria for alternative lengthening of telomeres by terminal restriction fragment length was that previously described (mean 
terminal restriction fragment length $>16 \mathrm{~kb}$, with a wide range in terminal restriction fragment lengths $<3$ to $>50 \mathrm{~kb}$ ). ${ }^{4}$ The terminal restriction fragment length and telomeric repeat amplification protocol assays were performed on frozen tumor samples.

\section{RNA-Seq}

A set of 12 glioblastoma (including seven non-defined telomere maintenance mechanism, two alternative lengthening of telomeres and three telomerase-positive tumors) and one telomerasepositive grade 3 oligodendroglioma were analyzed for gene expression using RNA-seq. Total RNA was isolated using the RNeasy Plus Mini Kit (Qiagen, Limburg, The Netherlands) and submitted for RNA sequencing (transcriptome) using the Illumina HiSeq 2000 (San Diego, CA, USA) at BGI Tech solutions (Tai Po, Hong Kong). mRNA was enriched using magnetic beads with oligo(dT), mRNA was fragmented and cDNA synthesized. Suitable sized fragments were selected using agarose gel electrophoresis and amplified using PCR. The quantity and quality of the sample libraries were evaluated using the 2100 Agilent Bioanaylzer (Agilent Technologies, Santa Clara, CA, USA) and ABI StepOnePlus Real-Time System (Life Technologies, Carlsbad, CA, USA) prior to sequencing. The bioinformatics analysis was also performed by BGI Tech solutions. Clean reads (at least 4G) were aligned to a human reference (ftp://ftp.ncbi.nih.gov/refseq/H_sapiens/mRNA

Prot/human.rna.fna.gz) sequence using SOA aligner/ SOAP2. ${ }^{31}$ Information provided included gene coverage and coverage depth. Alignment results that passed quality control proceeded to downstream analyses. The transcriptional levels of annotated genes are represented as fragments per kilobase of transcript per million mapped reads. Differential expression analysis between tumors was analyzed using Gene Ontology (GO) enrichment and Pathway enrichment (KEGG) analysis.

\section{Quantitative PCR}

Total RNA (500 ng) was extracted using Trizol DNase-treated, and cDNA synthesized using oligo-dT primers and SuperScriptIII (Life Technologies). Quantitative real-time PCR reactions were performed in triplicate using an ABI 7300 Real-Time PCR System and SYBR Green PCR Master Mix (Life Technologies). The comparative $C_{\mathrm{T}}$ method was used for the comparative quantification of gene expression, with results from each time point normalized against glyceraldehyde-3-phosphate dehydrogenase (GAPDH), and expressed as the fold difference over that in human brain (Clontech, Takara Bio, Otsu, Japan). Primer sequences were those reported previously. ${ }^{32-35}$

\section{Western Blotting}

Equal amounts of each protein lysate were separated on $4-12 \%$ NuPAGE Novex Bis-Tris Mini Gels (Life Technologies). Blots were incubated with a CD163 antibody (EPR14643; Abcam). Alkaline phosphatase secondary antibodies were detected using the WesternBreeze Chemiluminescent Kit detection system according to the manufacturer's instructions (Life Technologies), and images were captured on X-ray film.

\section{Statistical Analysis}

RNA-Seq and quantitative PCR data are expressed as the mean and s.d. For comparison of RNA-Seq data between tumor subgroups, mean fragments per kilobase of transcript per million mapped reads values were compared using independent $t$-tests. The false discovery rate was controlled by adjusting the $P$-values using the Benjamini-Hochberg algorithm (false discovery rate-adjusted $P$-value $<0.05$ was taken as a significant difference). Differences in quantitative PCR were investigated using one-way analysis of variance (ANOVA) followed by Tukey's multiple comparisons test, using GraphPad Prism software version 6.00 for Macintosh (GraphPad Software, San Diego, CA, USA). The frequency of tumor-associated macrophages among glioblastoma subgroups was investigated using the $\chi^{2}$ test. Kaplan Meier survival curves were compared between groups using the log-rank test using SPSS version 22 software, with $P$-values corrected for multiple comparisons using the Bonferroni correction. $P<0.05$ was considered statistically significant.

\section{Results}

\section{RNA-Seq Identified an Immune Signature in Non- Defined Telomere Maintenance Mechanism Tumors}

Representative tumors from the non-defined telomere maintenance mechanism subgroup were sequenced using RNA-Seq. The tumors sequenced included four non-defined telomere maintenance mechanism tumors that were negative and three that were positive for alternative lengthening of telomeresassociated promyelocytic leukemia bodies. Two alternative lengthening of telomeres glioblastomas, three telomerase-positive glioblastomas, and one grade 3 oligodendroglioma that was telomerase positive were also analyzed using RNA-seq for comparison. An analysis of gene expression differences between tumors with the non-defined telomere maintenance and other telomerase maintenance mechanisms using the Gene Ontology process database revealed immune signature pathways in the top 10 Gene Ontology terms altered. Immune system process (GO:0002376) was the third ranked Gene Ontology terms altered following system 
development (GO:0048731) and multicellular organismal development (GO:0007275). The top 10 Gene Ontology terms altered also included immune system process (GO:0002376) and regulation of immune system process (GO:0002682). An analysis of differentially expressed genes in non-defined telomere maintenance mechanism tumors identified many immune-based pathways including leukocyte trans-endothelial migration, hematopoietic cell lineage, and complement and coagulation cascades.

Of the immune cells present in glioblastoma antigen-presenting cells are the most difficult to definitively distinguish from malignant cells using morphology alone. Eight markers associated with macrophages and other antigen-presenting cells including CD163, CD68, CD14, MSRI (macrophage scavenger receptor 1), HLA-DRA, HLA-DRB1, $H L A-D P B 1$, and FCGR2A were selected and relative expression of each transcript (fragments per kilobase of transcript per million mapped reads values) compared between non-defined telomere maintenance mechanism and telomerase plus alternative lengthening of telomeres tumors combined. Non-defined telomere maintenance mechanism tumors had increased expression of each marker (Figure 1a, six markers false discovery rate-adjusted $P$-values $<0.01$, and two markers false discovery rate-adjusted $P$-values $<0.05)$. Non-defined telomere maintenance mechanism tumors also had increased expression of chemokines by macrophages (CXCL8), monocyte chemotactic proteins (CCL2), hematopoietic transcription factor (Spi-1 proto-oncogene, SPI1), and regulators of antigen presentation (CD74), false discovery rate-adjusted $P$-values $<0.05$; Figure $1 b$ ). Consistent with an increased immune signature many genes involved in the complement cascade were increased in non-defined telomere maintenance mechanism compared with telomerase plus alternative lengthening of telomeres tumors (Supplementary Figure S1). Expression data (fragments per kilobase of transcript per million mapped reads values) for a greater list of immune and other differentially expressed genes of interest for individual tumors are given in Supplementary Table S1.

\section{Non-Defined Telomere Maintenance Mechanism Glioblastoma had Increased CD163-Positive Tumor-Associated Macrophages}

The RNA-Seq data suggested that non-defined telomere maintenance mechanism tumors have increased numbers of antigen-presenting cells compared with alternative lengthening of telomeres and telomerase-positive tumors. To further
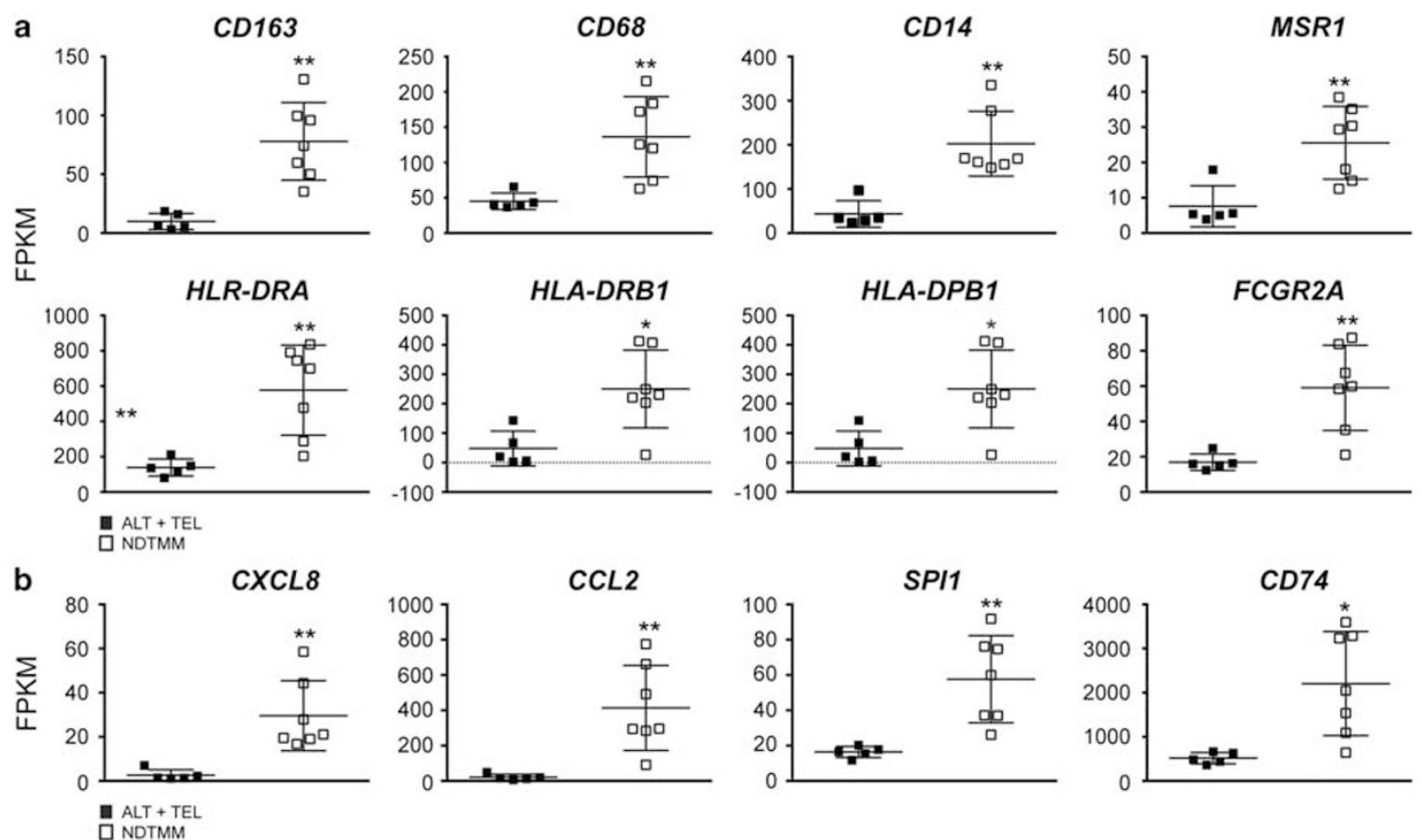

Figure 1 Increased antigen-presenting cell expression in non-defined telomere maintenance mechanism (NDTMM) glioblastoma from RNA-Seq data. Glioblastomas with different telomere maintenance mechanisms were sequenced using RNA-Seq. (a) NDTMM tumors had increased expression of selected antigen-presenting cell markers compared with telomerase (TEL) and alternative lengthening of telomeres (ALT) glioblastoma. (b) NDTMM had increased gene expression for chemokines produced by macrophages (CXCL8), monocyte recruitment (CCL2), transcription factor that activates gene expression during myeloid development (Spi-1 Proto-Oncogene, SPI1), and chaperone that regulates antigen presentation for the immune response (CD74) compared with alternative lengthening of telomeres (ALT) and telomerase (TEL) glioblastoma. *The false discovery rate-adjusted $P$-value $<0.05 ;{ }^{*}$ The false discovery rate-adjusted $P$-value $<0.01$. Error bars, mean \pm s.d.; FPKM, fragments per kilobase of transcript per million mapped reads. 
investigate this, the haptoglobin-hemoglobin (Hp-Hv) complex receptor, CD163, was selected for immunohistochemisty as it was increased in non-defined telomere maintenance mechanism tumors by RNA-Seq. The CD163 marker is also exclusively expressed on cells of monocyte/macrophage lineage and activated microglia, and is a key marker of M2 macrophages thought to promote tumorigenesis in glioblastoma. ${ }^{25,36-38} \mathrm{~A}$ high content of CD163positive cells was identified in $36 \%$ (49/136) of glioblastoma using immunohistochemistry. In relation to telomere maintenance mechanism subtype, a high content of CD163-positive cells were found in $79 \%(45 / 57)$ of the non-defined telomere maintenance mechanism group, $2 \%(1 / 50)$ of telomerase-positive tumors, and $14 \% \quad(4 / 29)$ of alternative lengthening of telomeres-positive tumors $(P<0.0001$, non-defined telomere maintenance mechanism compared with alternative lengthening of telomeres and telomerase-positive tumors).

All non-defined telomere maintenance mechanism tumors positive for alternative lengthening of telomeres-associated promyelocytic leukemia bodies, but negative for long and heterogeneous telomere lengths had an increased content of tumor-associated macrophages. This suggested that the increased tumor-associated macrophages in non-defined telomere maintenance mechanism tumors may affect the assignment of the alternative lengthening of telomeres telomere maintenance mechanism using the terminal restriction fragment length assay. The assignment of telomerase activity may also be affected with an increased content of tumorassociated macrophages. Expression of hTERT was increased in non-defined telomere maintenance mechanism tumors without alternative lengthening of telomere-associated promyelocytic leukemia bodies (fragments per kilobase of transcript per million mapped reads $0.18,0.17,0.35,0.84$ ) compared with alternative lengthening of telomeres and non-defined telomere maintenance mechanism tumors with alternative lengthening of telomeresassociated promyelocytic leukemia bodies (hTERT, fragments per kilobase of transcript per million mapped reads values $\leq 0.001$; Supplementary Table S1). All non-defined telomere maintenance mechanism tumors without alternative lengthening of telomeres-associated promyelocytic leukemia bodies were positive for PAX8 using immunohistochemistry (data not shown) consistent with a previous report in which non-defined telomere maintenance mechanism tumors were predominantly PAX8 positive. ${ }^{28}$ The increased hTERT by RNA-Seq and the PAX8-positive status of these tumors suggest non-defined telomere maintenance mechanism tumors with tumor-associated macrophages, but without alternative lengthening of telomeresassociated promyelocytic leukemia bodies, may have malignant cells that are telomerase positive. However, this was not directly assessed.
Further evidence that telomerase positive and nondefined telomere maintenance mechanism tumors without alternative lengthening of telomeresassociated promyelocytic leukemia bodies may be similar was evident from the RNA-Seq data (Supplementary Figure S2). Both tumor types had increased expression of PLK2 (polo-like kinase 2), PARVA (parvin alpha), MSN (moesin), MT1L (metallothionein 1, gene/pseudogne), MT1M (metallothionein 1M), CA9 (carbonic anhydrase IX), and MEOX2 (mesenchyme homeobox 2).

Based on the assumption that the presence of increased numbers of tumor-associated macrophages would affect the assignment of telomere maintenance mechanisms by telomeric repeat amplification protocol assay or terminal restriction fragment length measurements the remainder of the study investigated clinico-demographic and other differences based on five alternative telomere maintenance subgroups as defined by molecular markers. The new subgroups were based on whether the tumor was alternative lengthening of telomeres or non-alternative lengthening of telomeres positive, and whether the tumor had a high or low content of tumor-associated macrophages. These groups were: alternative lengthening of telomeres, for an alternative lengthening of telomeresassociated promyelocytic leukemia body positive tumor with a low content of CD163-positive cells; alternative lengthening of telomeres with macrophages, for an alternative lengthening of telomeres-associated promyelocytic leukemia body positive tumor with a high content of CD163-positive cells; telomerase positive for an hTERT and telomerase-positive tumor with a low content of CD163-positive cells; and telomerase positive with macrophages for a non-defined telomere maintenance mechanism tumor with hTERT and a high content of CD163-positive cells. These four groups encompassed 124 of the original $136(91 \%)$ glioblastomas used in this study. The fifth group contained 12 non-defined telomere maintenance mechanism tumors with a low content of macrophages (non-defined telomere maintenance mechanism-revised).

The increased amount of CD163-positive cells in alternative lengthening of telomeres with macrophages compared with alternative lengthening of telomeres tumors is shown in Figure 2a and b, and that for telomerase with macrophages compared with telomerase tumors in Figure $3 \mathrm{a}$ and $\mathrm{b}$. Western blotting was used to confirm increased CD163 protein in alternative lengthening of telomeres with macrophages and telomerase with macrophages tumors compared with alternative lengthening of telomeres and telomerase (alternative lengthening of telomeres with macrophages and alternative lengthening of telomeres data not shown, telomerase with macrophages and telomerase-positive tumors; Figure 3c). 


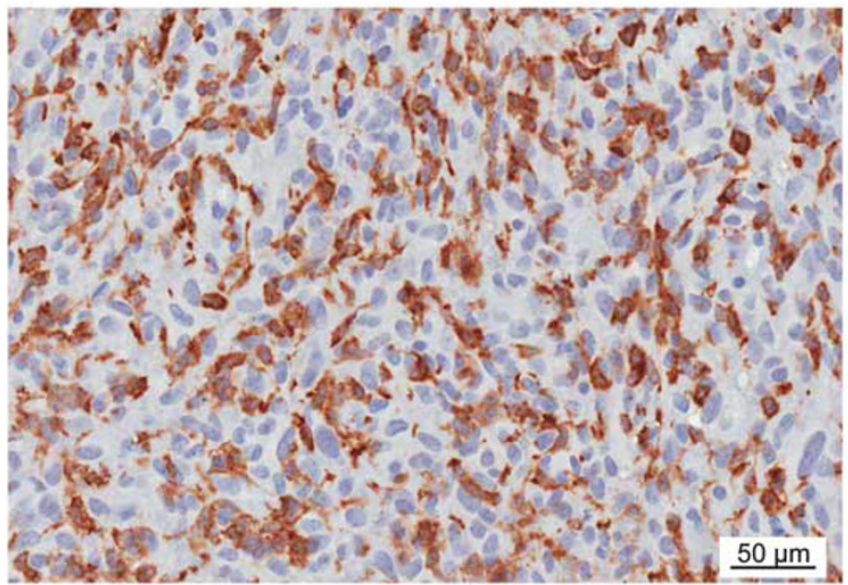

Increased CD163 staining in ALTM glioblastoma

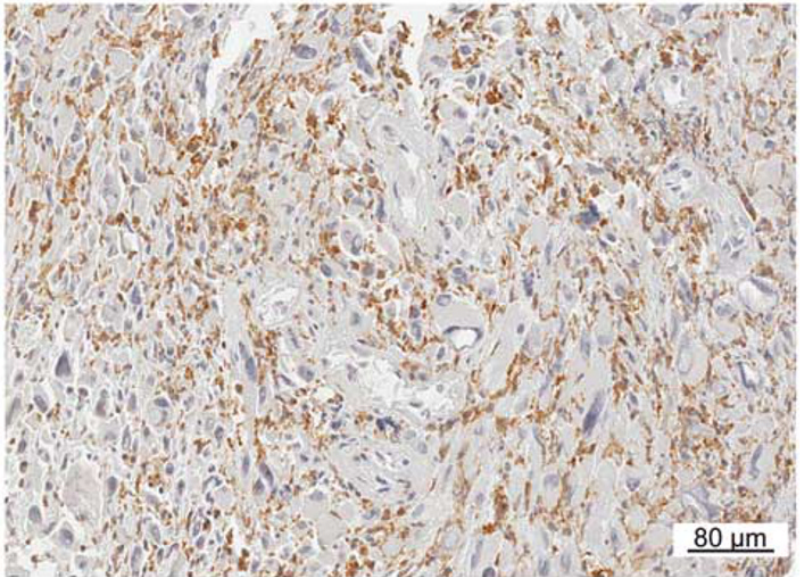

b

Decreased CD163 staining in ALT glioblastoma
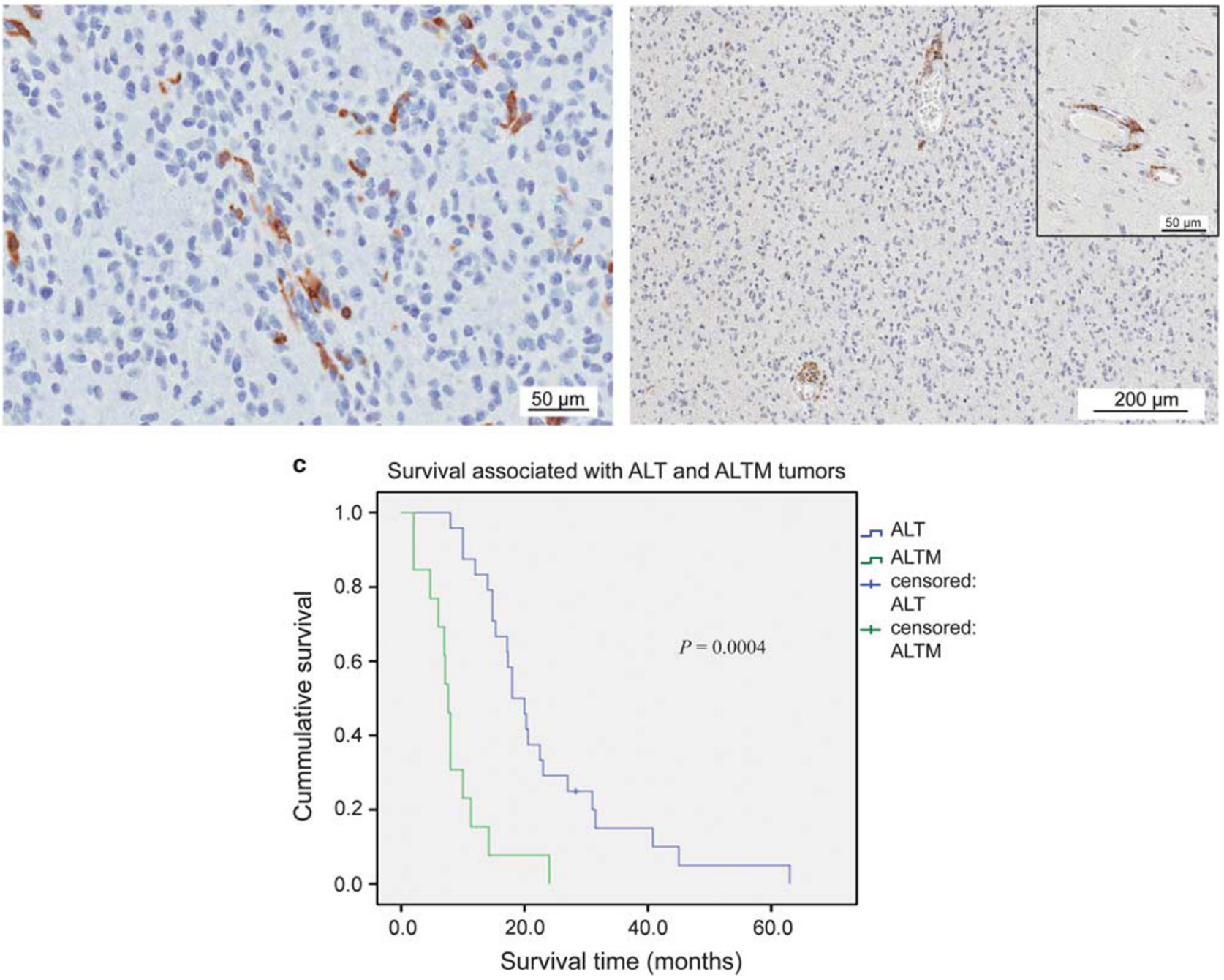

Figure 2 Increased CD163-positive cells and poorer patient survival associated with alternative lengthening of telomeres with macrophages glioblastoma. (a) Examples of CD163 staining to illustrate tumors with increased CD163-positive cells in alternative lengthening of telomeres (ALT) tumors with increased macrophage/microglia gene signature by RNA-Seq. (b) Examples of CD163 staining in alternative lengthening of telomeres tumors without a macrophage/microglia gene signature by RNA-Seq to illustrate reduced CD163positive cells. Inset: CD163-positive cells were found around blood vessels as part of the blood-brain barrier were used as a positive control. (c) An increased macrophage/microglia content in alternative lengthening of telomere-positive tumors was associated with poorer patient survival. ALT, alternative lengthening of telomeres tumors with reduced tumor-associated macrophages; ALTM, alternative lengthening of telomeres tumors with a high content of tumor-associated macrophages. 


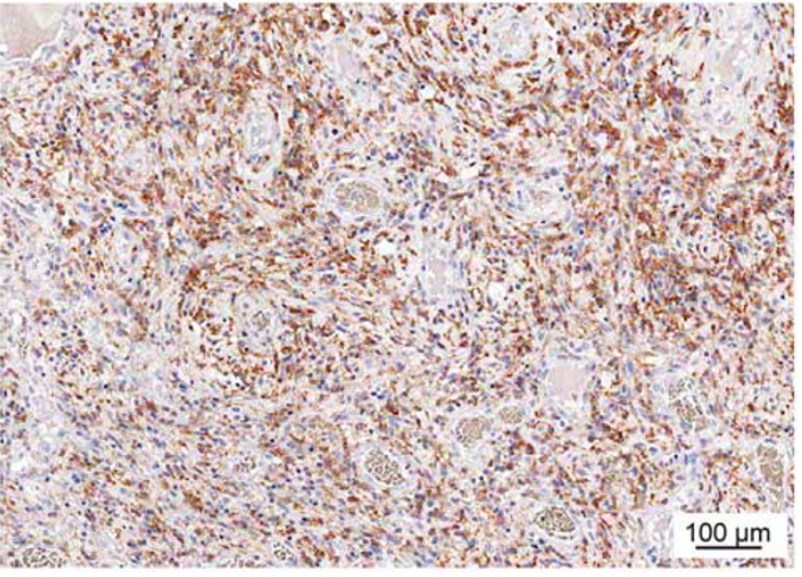

b

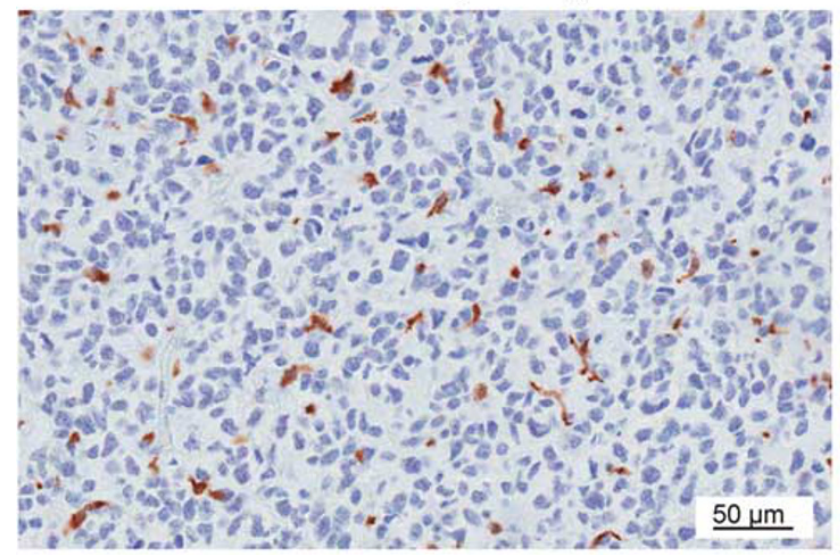

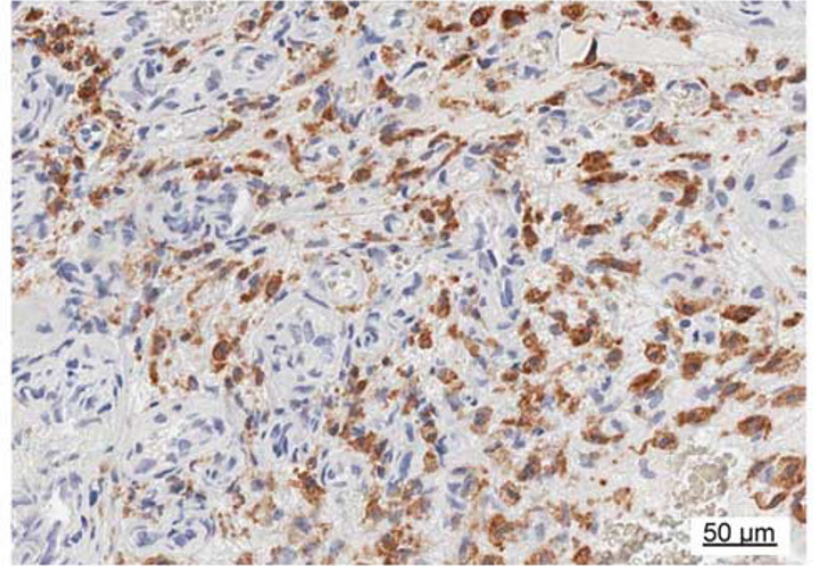

c

TEL

TELM
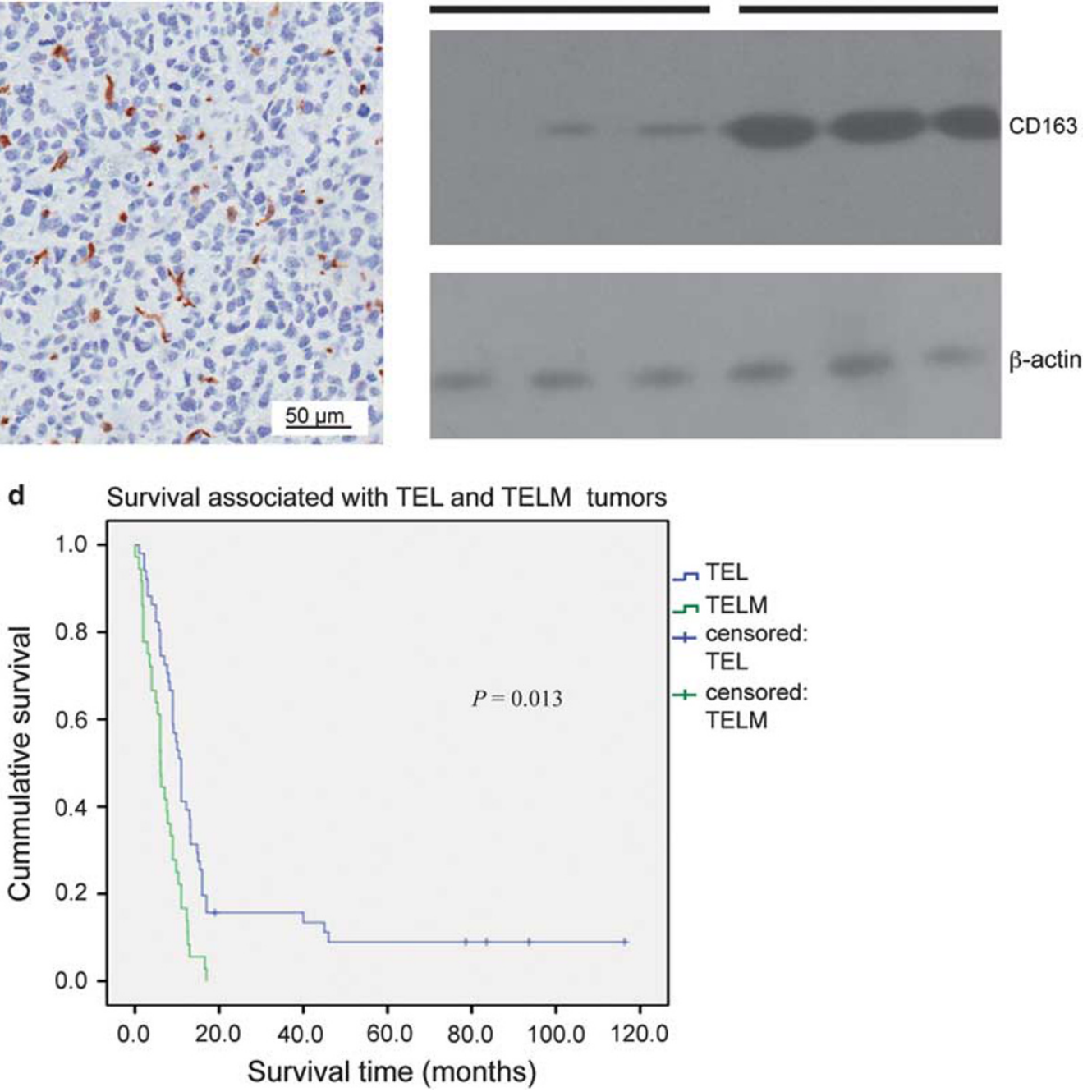

Figure 3 Increased CD163-positive cells and poorer patient survival associated with telomerase with macrophages glioblastoma. (a) Examples of CD163 staining to illustrate tumors with increased CD163-positive cells in telomerase with macrophages (TELM) tumors with a macrophage/microglia gene signature by RNA-Seq. (b) An Example of CD163 staining in telomerase (TEL) tumors without a macrophage/ microglia gene signature by RNA-Seq to illustrate reduced CD163-positive cells. (c) Western blotting to illustrate increased CD163 protein in telomerase with macrophages compared with telomerase tumors. $\beta$-Actin, loading control. (d) An increased tumor-associated macrophages content in telomerase with macrophages tumors was associated with poorer patient survival. TEL, telomerase positive with a low content of tumor-associated macrophages; TELM, non-defined telomere maintenance mechanism tumor without alternative lengthening of telomeres-associated promyelocytic leukemia bodies that is potentially telomerase positive with a high content of tumorassociated macrophages. 
Table 1 Clinico-demographic parameters for the five glioblastoma subgroups

\begin{tabular}{|c|c|c|c|c|c|}
\hline & $A L T$ & ALTM & $T E L$ & TELM & NDTMM-revised \\
\hline Total number of cases & 24 & 13 & 51 & 36 & 12 \\
\hline Median age (years) & $38(31.5-60)$ & $48(38-65.5)$ & $60(54-67)$ & $64(58-70.75)$ & $66.5(58.9-71.1)$ \\
\hline Median survival (months) & $18^{*}(14.41-21.59]$ & $7.66(6.78-8.54]$ & $11^{\#}(9.43-12.57)$ & $6.05(5.22-6.88)$ & $3.7(2-5.4)$ \\
\hline Surgery only & 0 & $3 \%(n=2)$ & $3.5 \%(n=12)$ & $2.5 \%(n=14)$ & $3 \%(n=9)$ \\
\hline Surgery+radiotherapy & $17.6 \%(n=12)$ & $8 \%(n=7)$ & $11 \%(n=19)$ & $6 \%(n=8)$ & $4.1 \%(n=3)$ \\
\hline Surgery+radiotherapy+TMZ & $20.3 \%(n=12)$ & $10.7 \%(n=4)$ & $16.5 \%(n=20)$ & $10.5 \%(n=14)$ & 0 \\
\hline$>24$ months survival & $\begin{array}{c}29 \%(n=7) \\
4 \text { treated with } \\
\text { TMZ }\end{array}$ & 0 & $\begin{array}{c}12 \%(n=6) \\
\text { all treated with } \\
\text { TMZ }\end{array}$ & 0 & 0 \\
\hline Near total/total resection & $79 \%(n=19)$ & $77 \%(n=10)$ & $80 \%(n=41)$ & $45 \%(n=16)$ & $75 \%(n=9)$ \\
\hline Partial resection /biopsy combined no. & $21 \%(n=5)$ & $23 \%(n=3)$ & $20 \%(n=10)$ & $55 \%{ }^{\S}(n=20)$ & $25 \%(n=3)$ \\
\hline partial: no. biopsy & $5: 0$ & $2: 1$ & $7: 3$ & $14: 6$ & $2: 1$ \\
\hline Frontal/temporal location & $71 \%(n=17)$ & $100 \%(n=13)$ & $57 \%(n=29)$ & $53 \%(n=19)$ & $83 \%(n=10)$ \\
\hline Parietal/occipital location & $29 \%(n=7)$ & 0 & $41 \%(n=21)$ & $42 \%(n=15)$ & $13 \%(n=2)$ \\
\hline Other location & 0 & 0 & $2 \%(n=1)$ & $6 \%(n=2)$ & 0 \\
\hline
\end{tabular}

Abbreviations: ALT, an alternative lengthening of telomeres-associated promyelocytic leukemia bodies positive tumor with a low content of tumorassociated macrophages; ALTM, an alternative lengthening of telomere-associated promyelocytic leukemia bodies positive tumor with a high content of tumor-associated macrophages; NDTMM-revised, a non-defined telomere maintenance mechanism tumor, without alternative lengthening of telomeres-associated promyelocytic leukemia bodies and without increased tumor-associated macrophages; TEL, a telomerasepositive tumor with a low content of tumor-associated macrophages; TELM, an alternative lengthening of telomeres-associated promyelocytic leukemia bodies negative tumor that is potentially telomerase positive with increased tumor-associated macrophages; TMZ, temozolomide; ${ }^{*} P<0.05$, ALT vs ALTM; ${ }^{P}>0.05$, TEL vs TELM; $\$ P<0.05$, TELM compared with ALTM, ALT, and TEL.

\section{Alternative Lengthening of Telomeres with Macrophages and Telomerase with Macrophages were Associated with Poorer Patient Survival}

The clinico-demographic features associated with the five glioblastoma subgroups are given in Table 1. No difference in patient age was found between alternative lengthening of telomeres and alternative lengthening of telomeres with macrophages, or telomerase and telomerase with macrophages subgroups. However, patient age was significantly decreased in alternative lengthening of telomeres and alternative lengthening of telomeres with macrophages compared with telomerase and telomerase with macrophages. This is consistent with a younger age for those with alternative lengthening of telomere-positive tumors. ${ }^{3}$ The alternative lengthening of telomeres with macrophages group had a median survival of 7.66 months (95\% CI, 6.78-8.54) that was reduced compared with the 18 months median survival of the alternative lengthening of telomeres group (95\% CI, 14.41-21.59; $P=0.0004$; Figure 2c). The telomerase with macrophages group had a median survival of 6.05 months (95\% CI, 5.22-6.88) that was reduced compared with the 11 months median survival of the telomerase group (95\% CI, 9.43-12.57; $P=0.013$; Figure 3d).

Survival for those with alternative lengthening of telomeres and alternative lengthening of telomeres with macrophages tumors had no significant effect on overall survival with radiotherapy (risk ratio, 0.42; 95\% CI, 6.78-8.54; $P=0.1$ ) or temozolomide treatment (risk ratio, 0.55; 95\% CI, 0.27-1.14; $P=0.44)$. Survival for those with telomerase and telomerase with macrophages tumors had a significant effect on overall survival with radiotherapy (risk ratio, $0.11 ; 95 \% \mathrm{CI}, 0.05-0.24 ; P<0.0001)$ and temozolomide treatment (risk ratio, $0.42 ; 95 \%$ CI, $0.26-0.79 ; P=0.021)$. Greater than 24 -month survival occurred for 13 individuals, all had either the alternative lengthening of telomeres subtype $(n=7)$ or the telomerase subtype $(n=6)$.

The extent of surgical resection could have contributed to the poorer outcome in the telomerase with macrophages subgroup. Fifty-five percent of the telomerase with macrophages group had only a partial resection or biopsy compared with $20 \%$ of telomerase, $23 \%$ of alternative lengthening of telomeres with macrophages, and $21 \%$ of alternative lengthening of telomeres subtype $(P=0.014)$. The location of the tumor was associated with survival for the telomerase group with poorer survival associated with tumors in the occipital and parietal lobe compared with tumors in the frontal and temporal lobe $(P=0.027$, median survival 10 vs 12.2 months).

The non-defined telomere maintenance mechanism-revised group had the poorest median survival (3.7 months). These tumors were not represented in the RNA-Seq analysis. An analysis of other markers tested in the cohort revealed all these tumors were MGMT positive (Supplementary Figure S3).

\section{Differences in Immune Signature Existed Between Alternative Lengthening of Telomeres with Macrophages and Telomerase with Macrophages Tumors}

Differences between the immune signatures of alternative lengthening of telomeres with macrophages and telomerase with macrophages tumors were investigated. In the RNA-Seq data alternative 


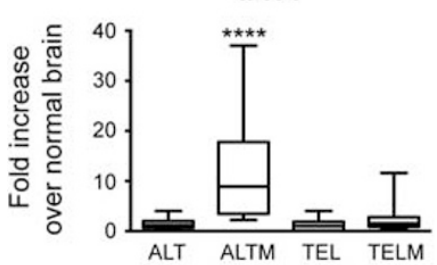

b

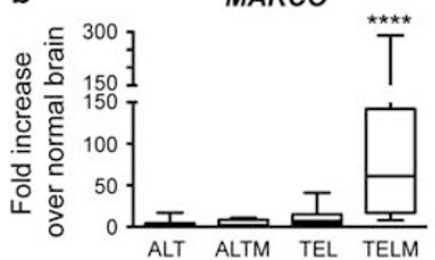

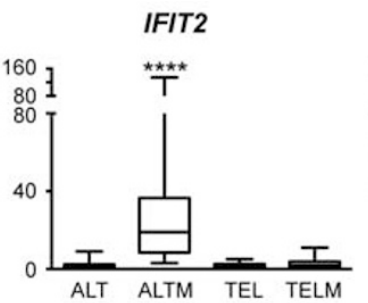

SRPX2

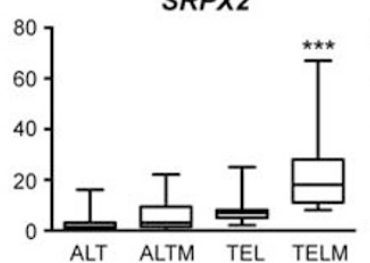

IFIT3

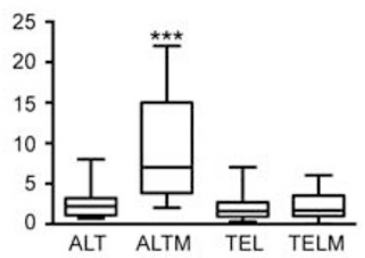

CXCL12

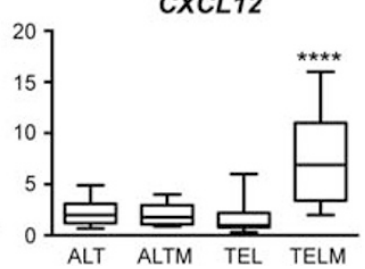

Figure 4 The immune signature in alternative lengthening of macrophages and telomerase with macrophages was different. (a) Real-time quantitative PCR confirmed alternative lengthening of telomeres with macrophages (ALTM) tumors had increased expression of 3 interferon-induced protein with tetratricopeptide (IFIT) genes (1, 2, and 3) compared with telomerase with macrophages (TELM); alternative lengthening of telomeres (ALT), and telomerase (TEL) tumors. (b) Real-time quantitative PCR confirmed telomerase with macrophages tumors had increased expression of the scavenger receptor macrophage receptor with collagenous structure (MARCO), sushirepeat containing protein, X-linked 2 (SRPX2), and CXCL12 compared with ALTM, TEL, and ALT tumors. Results mean, with the highest and lowest values included. ${ }^{*} * P<0.001 ; * * * * P<0.0001$. Tumor subgroups: ALT, an alternative lengthening of telomeres-associated promyelocytic leukemia body positive tumor with a low content of tumor-associated macrophages; ALTM, an alternative lengthening of telomeres-associated promyelocytic leukemia body positive tumor with a high content of tumor-associated macrophages; TEL a telomerase-positive tumor with a low content of tumor-associated macrophages; TELM, non-defined telomere maintenance mechanism tumor without alternative lengthening of telomeres-associated promyelocytic leukemia bodies that is potentially telomerase positive with a high content of tumor-associated macrophages.

lengthening of telomeres with macrophages had increased expression of interferon-induced proteins with tetratricopeptide repeats (IFIT1, IFIT2, and IFIT3) compared with telomerase with macrophages tumors (Supplementary Table S1). Increased IFIT1, IFIT2, and IFIT3 in telomerase with macrophages compared with the other subgroups was confirmed using real-time quantitative PCR $\quad(P<0.001$; Figure 4a).

The immune signature was strongest in telomerase with macrophages tumors in relation to the number of genes increased (Supplementary Table S1) and these included CCL18, CXCL2, CXCL12, CXCL14, MARCO, SAA1 (serum amyloid A), TLR (tol1-like receptor) 1, TLR4, TLR5, TLR6, TLR, and SRPX2. For the telomerase with macrophages group expression levels of three genes, MARCO, SRPX2, and CXCL12, were confirmed by quantitative PCR. All three genes were increased in telomerase with macrophages compared with the other subgroups $(P<0.001$; Figure $4 b)$.

\section{Discussion}

This study has shown for the first time that the majority of glioblastomas without a defined telomere maintenance mechanism contain a high content of tumor-associated macrophages. This has led us to propose that an increased tumor-associated macrophage content affects attempts to identify alternative lengthening of telomeres and telomerase activity.
Defined markers, based on the results from RNA-seq, allowed us to reclassify the non-defined telomere maintenance mechanisms into three subgroups. One subgroup, alternative lengthening of telomeres with macrophages, contained alternative lengthening of telomeres like tumors with increased numbers of macrophages. The second subgroup, telomerase with macrophages, contained telomerase like tumors with increased macrophages. The third subgroup may represent a true non-defined telomere maintenance mechanism group with low numbers of macrophages and for which a telomere maintenance mechanism has not been described. This study also identified differences in the immune signature between alternative lengthening of telomeres with macrophages and telomerase with macrophages tumors, suggesting different tumor-associated macrophages may associate with different telomere maintenance mechanisms in glioblastoma.

An increased content of tumor-associated macrophages is associated with poorer prognosis in many cancers, ${ }^{39}$ and increased tumor-associated macrophages contribute to a poorer outcome from high-grade glioma. ${ }^{25,40}$ Many properties of tumorassociated macrophages promote tumorigenesis. Invasion of glioma-like stem cells is enhanced by tumor-associated macrophages, ${ }^{41}$ and increased IL-10 from tumor-associated macrophages increases glioma cell proliferation. ${ }^{42}$ An added issue with tumor-associated macrophages is their lack of phagocytic and antigen-presenting activities and their inability to produce an effective immune 
activation against malignant cells, ${ }^{43-45}$ consistent with a role for tumor-associated macrophages in promoting rather than impairing tumor development. A significant contributing factor to the pro-tumorigenic function of tumor-associated macrophages is their strong M2 'alternatively activated macrophage' characteristics. ${ }^{25,46}$ Co-transplantation of M2 tumor-associated macrophages and glioma stem cells enhanced glioma cell growth. ${ }^{38}$

In the current study the increased CD163-positive tumor-associated macrophages in alternative lengthening of telomeres with macrophages and telomerase with macrophages was consistent with these tumors containing an increased content of M2 macrophages. The M2 immune signature appears strongest in the telomerase with macrophages subgroup with enhanced M2 markers including CXCL12 and $M A R C O$, which were absent in alternative lengthening of telomeres with macrophages tumors. To the best of our knowledge MARCO has not been investigated in tumor-associated macrophages in glioblastoma. The MARCO receptor binds environmental particles, bacteria, and oxidized lipids. ${ }^{33,47-49}$ The absence of MARCO leads to an increased inflammatory response suggesting MARCO modulates the immune response. ${ }^{50,51}$ In lung alveolar macrophages increased MARCO expression polarized lung alveolar macrophages toward a profibrotic M2 phenotype. ${ }^{52}$ However, MARCO does not exclusively exist on M2 macrophages being associated with M1 and dendritic cell activation. ${ }^{53-55}$ Increased CXCL12 promotes neo-angiogenesis, and supported by increased expression of SRPX2, may also mediate angiogenesis providing further evidence that tumorassociated macrophages in telomerase with macrophages are more tumor-promoting compared with those in alternative lengthening of telomeres with macrophages. ${ }^{56,57}$ Telomerase with macrophage tumors were associated with a reduced extent of surgical resection, suggesting tumor-associated macrophages in telomerase with macrophages are more infiltrative and thus damaging.

Tumor-associated macrophages contribute to resistance to radiotherapy and chemotherapy. ${ }^{58}$ In the current study an association with improved survival was not found with the addition of temozolomide for those with alternative lengthening of telomerepositive tumors making it difficult to ascertain if the presence of tumor associated with macrophages in alternative lengthening of telomeres with macrophages tumors contributed to treatment resistance. Prior to the introduction of temozolomide those with telomerase positive and non-defined telomere maintenance mechanism tumors had similar overall survival that was poorer in comparison to those with alternative lengthening of telomeres-positive tumors. ${ }^{3}$ Although telomerase and telomerase with macrophages tumors were both associated with improved survival with temozolomide, the advantages of temozolomide may be greatest for those without a high content of tumor-associated macrophages, particularly longer-term survival. Prior to temozolomide treatment, patients surviving longer than 2 years were solely comprised of those with alternative lengthening of telomeres-positive tumors. ${ }^{3}$ This is no longer the case as individuals with telomerase tumors achieve longer-term survival with temolozomide treatment. Despite the introduction of temolozomide longer-term survival has not emerged for those with telomerase with macrophages tumors. However, the reduced frequency of total/near total resections in telomerase with macrophages tumors may be a contributing factor to a poorer response to temozolomide. According to Geisenberger et al, ${ }^{59}$ long-term survivors had an M1 macrophage compared with short-term survivors that had an M2 macrophage gene signature. ${ }^{59}$ In additional to an altered immune signature, the same study found that long-term survivors were distinguished by a concurrent gain of chromosomes 19 and 20.59

Targeting M2 macrophages by removing them or switching them to a proinflammatory phenotype are potential new strategies for treatment. In mouse models of cervical and mammary tumors inhibition of the colony-stimulating factor 1 receptor (CSF-1R) decreased tumor-associated macrophages and enhanced tumor infiltration of cytotoxic $\mathrm{T}$ cells sufficient to inhibit tumor growth. ${ }^{60}$ Treatment of proneural glioblastoma with a CSF-1R inhibitor reduced M2 macrophages and tumor growth in xenografts. ${ }^{61}$ Reducing tumor-associated macrophages in telomerase with macrophages tumors may produce an improved sensitivity to temozolomide. This assumption is stronger if the malignant cells in telomerase and telomerase with macrophage tumors are also similar. Based on results from the current and previous studies this appears to be the case. The majority of telomerase and telomerase with macrophages tumors are PAX8 positive. ${ }^{28}$ Considering $P A X 8$ enhances telomerase activity, telomerase with macrophages tumors would be expected to be telomerase positive. ${ }^{29}$ An increased content of nonmalignant cells provided by tumor-associated macrophages provides one explanation for why telomerase activity was not detected in telomerase with macrophages tumors.

Consistent with telomerase with macrophages being telomerase positive, increased hTERT expression was found using RNA-Seq compared with alternative lengthening of telomeres and alternative lengthening of telomeres with macrophages tumors. However, the values to estimate the expression of hTERT were low $(<1)$ in telomerase with macrophages tumors. In telomerase-positive cell lines only a few copies of hTERT may be found and there is a correlation between telomerase activity and hTERT. ${ }^{62}$ Telomerase activity is also present in activated lymphocytes, so the increased hTERT in telomerase with macrophages may reflect nonmalignant cell activity. ${ }^{63}$ If this were the case hTERT would be expected to be increased in alternative 
lengthening of telomeres and alternative lengthening of telomeres with macrophages tumors wherein lymphocytes are occasionally found. Independent functions for hTERT exist, so the increased hTERT in telomerase with macrophages may not be associated with telomerase activity. ${ }^{64}$

Telomerase and telomerase with macrophages tumors had increased expression of other genes in comparison with alternative lengthening of telomeres and alternative lengthening of telomeres with macrophages tumors including genes involved in mineral absorption (MTIL, MTIM) and others (MSN, MEOX2, PARVA, PLK2, and CA9). However, telomerase tumors had increased expression of numerous HOX genes that were not found for telomerase with macrophages tumors (Supplementary Table S1). A greater comparison of the malignant cells in telomerase and telomerase with macrophages could be possible with the removal of tumor-associated macrophages to increase the proportion of malignant cells. This approach could also directly associate telomerase activity with telomerase with macrophages tumors.

In glioblastoma tumor-associated macrophages could originate from resistant microglia or from monocytes recruited from peripheral blood. ${ }^{38,65,66}$ Recent evidence suggests that the majority of tumor associated with macrophages in glioblastoma originate from peripheral monocytes in response to periostin (POSTN) produced from glioma stem cells. ${ }^{38}$ The immune signature in telomerase with macrophages and alternative lengthening of telomeres with macrophages showed distinctive differences, suggesting different subtypes of glioblastoma have different tumor-associated macrophages. Whether the tumor-associated macrophages in telomerase with macrophages and alternative lengthening of telomeres with macrophages tumors have the same origin is unknown. Alternative lengthening of telomeres with macrophages tumors are infrequent compared with telomerase with macrophages so they may not be captured in other studies.

Alternative lengthening of telomeres with macrophages tumors had increased expression of three of the four IFIT family genes. The reason for these genes being increased in an immune signature associated with alternative lengthening of telomeres is not obvious. The IFIT genes are interferon response genes best known for their increased expression following viral infection where they are thought to have immuno-modulatory activity ${ }^{67,68}$ These genes are also increased in response to other stimuli including interferon treatment, pathogen-associated molecular pattern (PAM) recognition, and retinoic acid. ${ }^{67,69}$ Although most cells do no express IFIT genes without stimuli, subsets of myeloid cells may ubiquitously express IFIT genes and these subsets may infiltrate alternative lengthening of telomeres with macrophages tumors. ${ }^{70}$

Whether increased tumor associated macrophages represent an advanced stage in the development of alternative lengthening of telomeres and telomerase tumors remains to be determined. A comparison of methylation levels in initial and recurrent tumors found an enrichment of macrophage activation genes in recurrent tumors, suggesting modulation of immune genes occurs in glioma progression. ${ }^{71}$ In the genome-based subtypes described by Phillips et al in 2006, ${ }^{13}$ it was proposed that the proneural and classical gliomas could progress to mesenchymal tumors. However, in the genome subtypes proposed by Verhaak et al in 2010 (ref. 14) recurrent tumors were the same subtype as the initial tumors suggesting tumor subtypes do not progress from one to another.

In the current study cohort seven individuals had recurrent tumors removed. The recurrent tumors comprised of three alternative lengthening of telomeres, four telomerase, and one telomerase with macrophages tumors. All recurrent tumors were the same subgroup as the initial tumor. However, with the exception of the telomerase with macrophages tumor, all tumors were from longer-term survivors with the recurrent tumor removed at least 1 year following the removal of the initial tumor. It is possible that tumors from longer-term survivors have mechanisms that prevent a large infiltration of tumor associated macrophages.

Glioblastomas that are alternative lengthening of telomeres positive are associated with IDH1 and TP53 mutations. ${ }^{2,4,12}$ If alternative lengthening of telomeres with macrophages were a progression from alternative lengthening of telomeres tumor a similar frequency of IDH1 and TP53 mutations would be expected. Only 15\% of the alternative lengthening of telomeres with macrophages group contained IDH1 mutations in contrast to $60 \%$ of the alternative lengthening of telomeres group, suggesting alternative lengthening of telomeres with macrophages is not a progression from alternative lengthening of telomeres. The difference in TP53 mutations was not significantly different $(46 \%$ of the alternative lengthening of telomeres with macrophages group had mutant TP53 compared with 58\% of the alternative lengthening of telomeres group). An increased content of tumor-associated macrophages may have affected the sensitivity for identifying TP53 and IDH1 mutations by DNA sequencing. Telomerase with macrophages and telomerase tumors had increased expression of the stable hypoxia marker CA9. ${ }^{72}$ Hypoxia is one factor that may recruit tumor associated macrophages. ${ }^{73}$ Therefore it is possible that hypoxia occurs first in telomerase glioblastomas leading to the increased recruitment of tumor associated macrophages.

The current study selected for an increased content of infiltrating macrophages that was uniformly distributed. Therefore a high content of tumor-associated macrophages limited to a small area of the tumor would not be accounted for in the current study and may still be important in predicting patient survival. Increased tumor-associated 
macrophages did not account for poor prognosis for all glioblastomas. The subset of non-defined telomere maintenance mechanism tumors without increased tumor-associated macrophages (non-defined telomere maintenance mechanism-revised) was associated with the worst median survival (3.7 months). These tumors are alternative lengthening of telomeres and telomerase activity negative, and MGMT positive. Greater detail on the molecular composition of these tumors is unknown as none of these tumors were represented in the RNA-Seq analysis. It is yet to be determined if these tumors have no telomere maintenance mechanisms or a third mechanism.

In summary, different immune signatures were present in alternative lengthening of telomeres and telomerase tumors, suggesting tumor-associated macrophages are different among tumor subtypes. An increased content of tumor-associated macrophages in alternative lengthening of telomeres and telomerase tumors could explain why previous attempts did not attribute a telomere maintenance mechanism to a significant proportion of glioblastomas. A high content of tumor-associated macrophages is associated with a poorer prognosis among telomere maintenance subtypes, and in telomerase tumors associated with a poorer surgical resection. New strategies that target tumor-associated macrophages may improve survival for different subgroups of glioblastoma.

\section{Acknowledgments}

Amanda Fisher, Janine Neill, and Alisha Shaw are thanked for technical expertise. Roslyn Kemp is thanked for scientific expertise. Neurosurgeons Reuben Johnson and Ronald Boet are thanked for contributing cases to the cohort. We are grateful to Dirk de Ridder for RNA-seq support, Helen Morrin and the Cancer Society Tissue Bank Christchurch, New Zealand. Funding for this study was provided by the Cancer Society New Zealand, Matariki Diagnostics, Dunedin School of Medicine, The Centre for Translational Cancer Research, and the Gut Health Network supported KWH.

\section{Disclosure/conflict of interest}

The authors declare no conflict of interest.

\section{References}

1 Stupp R, Mason WP, van den Bent MJ et al. Radiotherapy plus concomitant and adjuvant temozolomide for glioblastoma. N Engl J Med 2005;352:987-996.

2 Chen YJ, Hakin-Smith V, Teo M et al. Association of mutant TP53 with alternative lengthening of telomeres and favorable prognosis in glioma. Cancer Res 2006;66: 6473-6476.
3 Hakin-Smith V, Jellinek DA, Levy D et al. Alternative lengthening of telomeres and survival in patients with glioblastoma multiforme. Lancet 2003;361:836-838.

4 Royds JA, Al Nadaf S, Wiles AK et al. The CDKN2A G500 allele is more frequent in GBM patients with no defined telomere maintenance mechanism tumors and is associated with poorer survival. PloS One 2011;6: e26737.

5 Marian CO, Cho SK, McEllin BM et al. The telomerase antagonist, imetelstat, efficiently targets glioblastoma tumor-initiating cells leading to decreased proliferation and tumor growth. Clin Cancer Res 2010;16:154-163.

6 Ozawa T, Gryaznov SM, Hu LJ et al. Antitumor effects of specific telomerase inhibitor GRN163 in human glioblastoma xenografts. Neuro Oncol 2004;6: 218-226.

7 Heaphy CM, Subhawong AP, Hong SM et al. Prevalence of the alternative lengthening of telomeres telomere maintenance mechanism in human cancer subtypes. Am J Pathol 2011;179:1608-1615.

8 Henson JD, Reddel RR. Assaying and investigating alternative lengthening of telomeres activity in human cells and cancers. FEBS Lett 2010;584:3800-3811.

9 Henson JD, Hannay JA, McCarthy SW et al. A robust assay for alternative lengthening of telomeres in tumors shows the significance of alternative lengthening of telomeres in sarcomas and astrocytomas. Clin Cancer Res 2005;11:217-225.

10 Bryan TM, Englezou A, Dalla-Pozza L et al. Evidence for an alternative mechanism for maintaining telomere length in human tumors and tumor-derived cell lines. Nat Med 1997;3:1271-1274.

11 Bryan TM, Reddel RR. Telomere dynamics and telomerase activity in in vitro immortalised human cells. Eur J Cancer 1997;33:767-773.

12 McDonald KL, McDonnell J, Muntoni A et al. Presence of alternative lengthening of telomeres mechanism in patients with glioblastoma identifies a less aggressive tumor type with longer survival. J Neuropathol Exp Neurol 2010;69:729-736.

13 Phillips HS, Kharbanda S, Chen R et al. Molecular subclasses of high-grade glioma predict prognosis, delineate a pattern of disease progression, and resemble stages in neurogenesis. Cancer Cell 2006;9: $157-173$.

14 Verhaak RG, Hoadley KA, Purdom E et al. Integrated genomic analysis identifies clinically relevant subtypes of glioblastoma characterized by abnormalities in PDGFRA, IDH1, EGFR, and NF1. Cancer Cell 2010;17: 98-110.

15 Carro MS, Lim WK, Alvarez MJ et al. The transcriptional network for mesenchymal transformation of brain tumours. Nature 2010;463:318-325.

16 Slatter T, Gifford-Garner J, Wiles A et al. Pilocytic astrocytomas have telomere-associated promyelocytic leukemia bodies without alternatively lengthened telomeres. Am J Pathol 2010;177:2694-2700.

17 Tabori U, Vukovic B, Zielenska $\mathrm{M}$ et al. The role of telomere maintenance in the spontaneous growth arrest of pediatric low-grade gliomas. Neoplasia 2006;8: 136-142.

18 Chen Q, Ijpma A, Greider CW. Two survivor pathways that allow growth in the absence of telomerase are generated by distinct telomere recombination events. Mol Cell Biol 2001;21:1819-1827. 
19 Siddiqa A, Cavazos D, Chavez J et al. Modulation of telomeres in alternative lengthening of telomeres type I like human cells by the expression of Werner protein and telomerase. J Oncol 2012;2012:806382.

20 Gocha AR, Acharya S, Groden J. WRN loss induces switching of telomerase-independent mechanisms of telomere elongation. PloS One 2014;9:e93991.

21 Morantz RA, Wood GW, Foster M et al. Macrophages in experimental and human brain tumors. Part 2: Studies of the macrophage content of human brain tumors. J Neurosurg 1979;50:305-311.

22 Morimura T, Neuchrist C, Kitz K et al. Monocyte subpopulations in human gliomas: expression of $\mathrm{FC}_{\mathrm{C}}$ and complement receptors and correlation with tumor proliferation. Acta Neuropathol 1990;80:287-294.

23 Roggendorf W, Strupp S, Paulus W. Distribution and characterization of microglia/macrophages in human brain tumors. Acta Neuropathol 1996;92:288-293.

24 Sasai K, Nodagashira M, Nishihara H et al. Careful exclusion of non-neoplastic brain components is required for an appropriate evaluation of O6-methylguanine-DNA methyltransferase status in glioma: relationship between immunohistochemistry and methylation analysis. Am J Surg Pathol 2008;32: 1220-1227.

25 Komohara Y, Ohnishi K, Kuratsu J et al. Possible involvement of the M2 anti-inflammatory macrophage phenotype in growth of human gliomas. J Pathol 2008;216:15-24.

26 Hsu CY, Lin SC, Ho HL et al. Exclusion of histiocytes/ endothelial cells and using endothelial cells as internal reference are crucial for interpretation of MGMT immunohistochemistry in glioblastoma. Am J Surg Pathol 2013;37:264-271.

27 Robson EJ, He SJ, Eccles MR. A PANorama of PAX genes in cancer and development. Nat Rev Cancer 2006;6:52-62.

28 Hung N, Chen YJ, Taha A et al. Increased paired box transcription factor 8 has a survival function in glioma. BMC Cancer 2014;14:159.

29 Chen YJ, Campbell HG, Wiles AK et al. PAX8 regulates telomerase reverse transcriptase and telomerase RNA component in glioma. Cancer Res 2008;68:5724-5732.

30 Kim NW, Piatyszek MA, Prowse KR et al. Specific association of human telomerase activity with immortal cells and cancer. Science 1994;266:2011-2015.

$31 \mathrm{Li} \mathrm{R}, \mathrm{Yu} \mathrm{C}$, Li Y et al. SOAP2: an improved ultrafast tool for short read alignment. Bioinformatics 2009;25: 1966-1967.

32 Sarma SN, Kim YJ, Ryu JC. Gene expression profiles of human promyelocytic leukemia cell lines exposed to volatile organic compounds. Toxicology 2010;271: 122-130.

33 Baqir M, Chen CZ, Martin RJ et al. Cigarette smoke decreases MARCO expression in macrophages: implication in Mycoplasma pneumoniae infection. Respir Med 2008;102:1604-1610.

34 Gao Z, Zhang J, Bi M et al. SRPX2 promotes cell migration and invasion via FAK dependent pathway in pancreatic cancer. Int J Clin Exp Pathol 2015;8: 4791-4798.

35 Boudot A, Kerdivel G, Lecomte S et al. COUP-TFI modifies CXCL12 and CXCR4 expression by activating EGF signaling and stimulates breast cancer cell migration. BMC Cancer 2014;14:407.

36 Borda JT, Alvarez X, Mohan M et al. CD163, a marker of perivascular macrophages, is up-regulated by microglia in simian immunodeficiency virus encephalitis after haptoglobin-hemoglobin complex stimulation and is suggestive of breakdown of the blood-brain barrier. Am J Pathol 2008;172:725-737.

37 Madsen M, Moller HJ, Nielsen MJ et al. Molecular characterization of the haptoglobin.hemoglobin receptor CD163. Ligand binding properties of the scavenger receptor cysteine-rich domain region. J Biol Chem 2004;279:51561-51567.

38 Zhou W, Ke SQ, Huang Z et al. Periostin secreted by glioblastoma stem cells recruits M2 tumour-associated macrophages and promotes malignant growth. Nat Cell Biol 2015;17:170-182.

39 Bingle L, Brown NJ, Lewis CE. The role of tumourassociated macrophages in tumour progression: implications for new anticancer therapies. J Pathol 2002;196: 254-265.

40 Noorani IPG, Grundy PL, Sharpe G et al. Novel association between microglia and stem cells in human gliomas: A contributor to tumour proliferation? J Pathol: Clin Res 2015;1:67-75.

41 Ye XZ, Xu SL, Xin YH et al. Tumor-associated microglia/macrophages enhance the invasion of glioma stem-like cells via TGF-beta1 signaling pathway. J Immunol 2012;189:444-453.

42 Wagner S, Czub S, Greif M et al. Microglial/macrophage expression of interleukin 10 in human glioblastomas. Int J Cancer 1999;82:12-16.

43 Hao C, Parney IF, Roa WH et al. Cytokine and cytokine receptor mRNA expression in human glioblastomas: evidence of Th1, Th2 and Th3 cytokine dysregulation. Acta Neuropathol 2002;103:171-178.

44 Moffett JR, Els T, Espey MG et al. Quinolinate immunoreactivity in experimental rat brain tumors is present in macrophages but not in astrocytes. Exp Neurol 1997;144:287-301.

45 Hussain SF, Yang D, Suki D et al. The role of human glioma-infiltrating microglia/macrophages in mediating antitumor immune responses. Neuro Oncol 2006;8: 261-279.

46 Ludwig HC, Feiz-Erfan I, Bockermann V et al. Expression of nitric oxide synthase isozymes (NOS I-III) by immunohistochemistry and DNA in situ hybridization. Correlation with macrophage presence, vascular endothelial growth factor (VEGF) and oedema volumetric data in 220 glioblastomas. Anticancer Res 2000;20:299-304.

47 Arredouani MS, Franco F, Imrich A et al. Scavenger Receptors SR-AI/II and MARCO limit pulmonary dendritic cell migration and allergic airway inflammation. J Immunol 2007;178:5912-5920.

48 Arredouani MS, Palecanda A, Koziel H et al. MARCO is the major binding receptor for unopsonized particles and bacteria on human alveolar macrophages. J Immunol 2005;175:6058-6064.

49 Dahl M, Bauer AK, Arredouani $M$ et al. Protection against inhaled oxidants through scavenging of oxidized lipids by macrophage receptors MARCO and SR-AI/II. J Clin Invest 2007;117:757-764.

50 Thakur SA, Beamer CA, Migliaccio CT et al. Critical role of MARCO in crystalline silica-induced pulmonary inflammation. Toxicol Sci 2009;108: 462-471.

51 Ghosh S, Gregory D, Smith A et al. MARCO regulates early inflammatory responses against influenza: a useful macrophage function with adverse outcome. Am J Respir Cell Mol Biol 2011;45:1036-1044. 
52 Murthy S, Larson-Casey JL, Ryan AJ et al. Alternative activation of macrophages and pulmonary fibrosis are modulated by scavenger receptor, macrophage receptor with collagenous structure. FASEB J 2015;29:3527-3536.

53 Stoger JL, Gijbels MJ, van der Velden S et al. Distribution of macrophage polarization markers in human atherosclerosis. Atherosclerosis 2012;225:461-468.

54 Martinez FO, Sica A, Mantovani A et al. Macrophage activation and polarization. Front Biosci 2008;13: 453-461.

55 Kissick HT, Dunn LK, Ghosh S et al. The scavenger receptor MARCO modulates TLR-induced responses in dendritic cells. PloS One 2014;9:e104148.

56 Kryczek I, Lange A, Mottram P et al. CXCL12 and vascular endothelial growth factor synergistically induce neoangiogenesis in human ovarian cancers. Cancer Res 2005;65:465-472.

57 Miljkovic-Licina M, Hammel P, Garrido-Urbani S et al. Sushi repeat protein X-linked 2, a novel mediator of angiogenesis. FASEB J 2009;23:4105-4116.

58 Deininger $\mathrm{MH}$, Pater S, Strik $\mathrm{H}$ et al. Macrophage/ microglial cell subpopulations in glioblastoma multiforme relapses are differentially altered by radiochemotherapy. J Neurooncol 2001;55:141-147.

59 Geisenberger C, Mock A, Warta R et al. Molecular profiling of long-term survivors identifies a subgroup of glioblastoma characterized by chromosome 19/20 cogain. Acta Neuropathol 2015;130:419-434.

60 Strachan DC, Ruffell B, Oei Y et al. CSF1R inhibition delays cervical and mammary tumor growth in murine models by attenuating the turnover of tumor-associated macrophages and enhancing infiltration by CD8 T cells. Oncoimmunology 2013;2:e26968.

61 Pyonteck SM, Akkari L, Schuhmacher AJ et al. CSF-1R inhibition alters macrophage polarization and blocks glioma progression. Nat Med 2013;19: 1264-1272.

62 Yi X, Shay JW, Wright WE. Quantitation of telomerase components and hTERT mRNA splicing patterns in immortal human cells. Nucleic Acids Res 2001;29: 4818-4825.
63 Weng NP, Levine BL, June CH et al. Regulated expression of telomerase activity in human T lymphocyte development and activation. J Exp Med 1996;183: 2471-2479.

64 Qin Y, Guo H, Tang B et al. The non-reverse transcriptase activity of the human telomerase reverse transcriptase promotes tumor progression (review). Int J Oncol 2014;45:525-531.

65 Staudt ND, Jo M, Hu J et al. Myeloid cell receptor LRP1/ CD91 regulates monocyte recruitment and angiogenesis in tumors. Cancer Res 2013;73:3902-3912.

66 Ling EA, Wong WC. The origin and nature of ramified and amoeboid microglia: a historical review and current concepts. Glia 1993;7:9-18.

67 Sen GC, Sarkar SN. The interferon-stimulated genes: targets of direct signaling by interferons, doublestranded RNA, and viruses. Curr Top Microbiol Immunol 2007;316:233-250.

68 Diamond MS, Farzan M. The broad-spectrum antiviral functions of IFIT and IFITM proteins. Nat Rev Immunol 2013;13:46-57.

$69 \mathrm{Yu}$ M, Tong JH, Mao M et al. Cloning of a gene (RIG-G) associated with retinoic acid-induced differentiation of acute promyelocytic leukemia cells and representing a new member of a family of interferon-stimulated genes. Proc Natl Acad Sci USA 1997;94:7406-7411.

70 Daffis S, Samuel MA, Keller BC et al. Cell-specific IRF-3 responses protect against West Nile virus infection by interferon-dependent and -independent mechanisms. PLoS Pathog 2007;3:e106.

71 Alentorn A, Duran-Pena A, Malousi A et al. Differential gene methylation in paired glioblastomas suggests a role of immune response pathways in tumor progression. J Neurooncol 2015;124:385-392.

72 Potter C, Harris AL. Hypoxia inducible carbonic anhydrase IX, marker of tumour hypoxia, survival pathway and therapy target. Cell Cycle 2004;3: 164-167.

73 Lewis C, Murdoch C. Macrophage responses to hypoxia: implications for tumor progression and anticancer therapies. Am J Pathol 2005;167:627-635.

Supplementary Information accompanies the paper on Modern Pathology website (http://www.nature.com/ modpathol) 